\title{
Filoviral haemorrhagic fevers: A threat to Zambia?
}

\author{
Authors: \\ Katendi Changula ${ }^{1}$ \\ A.S. Mweene ${ }^{1}$ \\ Affiliations: \\ ${ }^{1}$ School of Veterinary \\ Medicine, University of \\ Zambia, Zambia \\ Correspondence to: \\ Katendi Changula \\ Email: \\ katendi.changula@sacids.org \\ Postal address: \\ PO Box 32379, Lusaka, \\ Zambia \\ How to cite this abstract: \\ Changula, K. \& Mweene, A.S., \\ 2012, 'Filoviral haemorrhagic \\ fevers: A threat to Zambia?', \\ Onderstepoort Journal of \\ Veterinary Research 79(2), \\ Art. \#465, 1 page. http:// \\ dx.doi.org/10.4102/ojvr. \\ v79i2.465 \\ Note: \\ Proceedings of the \\ Conference of the Southern \\ African Centre for Infectious \\ Disease Surveillance 'One \\ Health' held at the National \\ Institute for Communicable \\ Diseases, Johannesburg, July \\ 2011.
}

Filoviral haemorrhagic fevers (FVHF) are caused by agents belonging to Filoviridae family, Ebola and Marburg viruses. They are amongst the most lethal pathogens known to infect humans. Incidence of FVHF outbreaks are increasing, with affected number of patients on the rise. Whilst there has been no report yet of FVHF in Zambia, its proximity to Angola and Democratic Republic of Congo, which have recorded major outbreaks, as well as the open borders, increased trade and annual migration of bats between these countries, puts Zambia at present and increased risk. Previous studies have indicated bats as potential reservoir hosts for filoviruses. An increasing population with an increasing demand for resources has forced incursion into previously uninhabited land, potentially bringing them into contact with unknown pathogens, reservoir hosts and/or amplifying hosts. The recent discovery of a novel arenavirus, Lujo, highlights the potential that every region, including Zambia, has for being the epicentre or primary focus for emerging and re-emerging infections. It is therefore imperative that surveillance for potential emerging infections, such as viral haemorrhagic fevers be instituted. In order to accomplish this surveillance, rapid detection, identification and monitoring of agents in patients and potential reservoirs is needed. International co-operation is the strategy of choice for the surveillance and fight against emerging infections. Due to the extensive area in which filoviral infections can occur, a regional approach to surveillance activities is required, with regional referral centres. There is a need to adopt shared policies for the prevention and control of infectious diseases. There is also need for optimisation of currently available tests and development of new diagnostic tests, in order to have robust, highly sensitive and specific diagnostic tests that can be used even where there are inadequate laboratories and diagnostic services. 